\title{
Polychromatic speckle reduction in laser pico-projectors using stationary dual Hadamard diffusers
}

\author{
W. Thomas \\ whthomas@mtu.edu \\ C. Middlebrook \\ ctmiddle@mtu.edu
}

\author{
Electrical and Computer Engineering Department, Michigan Technological University, Houghton, MI \\ 49931, United States of America \\ Electrical and Computer Engineering Department, Michigan Technological University, Houghton, MI \\ 49931, United States of America
}

Speckle from coherent light sources continues to be a limiting factor in the design and manufacturing of laser projectors. Utilizing diffractive diffusers has been an effective method for reducing monochromatic speckle contrast. Color laser projection devices require the use of red, green, and blue laser sources therefore; using a monochromatic diffractive diffuser may not be optimal for color speckle contrast reduction. A new static dual-diffuser system utilizing Hadamard matrices has been designed to reduce full color speckle. This paper analyzes the effectiveness of the new Hadamard diffusers in reducing speckle using three separate RGB laser diodes. A simulation of the Hadamard diffusers is conducted to determine the optimum spacing between the two diffusers for polychromatic speckle reduction. Experimental measured results are presented using the optimal spacing of Hadamard diffusers for RGB color speckle reduction showing $60 \%$ reduction in contrast.

[DOI: http://dx.doi.org/10.2971/jeos.2014.14059]

Keywords: Polychromatic speckle, laser pico-projectors, speckle contrast reduction, Hadamard matrix, diffractive diffusers

\section{INTRODUCTION}

Handheld electronic device size continues to decrease while ever increasing in capabilities. One needed capability is a small stand alone or mobile phone integrated hand held picoprojector that can be used under general lighting conditions. Laser diodes can be easily integrated into electronic devices and are able to provide a bright high resolution image while providing a large color gamut. Laser light sources also allow for infinite focus reducing user setup. Laser diodes are not currently used in pico-projectors due to the introduction of speckle resulting from the highly coherent source.

Speckle is a granular irregularity in the laser image caused by the interference of the light reflected from a rough surface. The eye observes this interference pattern - speckle - as a visual disturbance leading to headaches or eye irritations [1]. The amount of speckle in an image is measured by the contrast. Speckle contrast, $C$, is calculated by dividing the standard deviation of image irradiance by the average image irradiance, I [2].

Diffractive diffusers have been investigated previously for use in speckle reduction applications but most analysis remains monochromatic [3]-[5]. In order to fully utilize diffusers in projector applications polychromatic speckle reduction is examined within this paper. Digital displays use a variation of only red, green, and blue colors for image projection. This research effort focuses on the three RGB wavelengths. Dual Hadamard matrix diffusers (DHDs) can be utilized for contrast reduction in polychromatic speckle reduction applications. Analysis of the DHDs ability to decrease speckle for each wavelength at 635,535 and $450 \mathrm{~nm}$ and in combination of all three wavelengths is presented in this paper. The polychromatic laser setup needed to measure the speckle contrast resulting from DHDs is also performed within this work. Full simulation and experimental results from these comprehensive analyses is presented. Finally, the distance of separation between the DHDs has been examined to determine the relationship between speckle contrast and spatial movement between two diffractive optical elements. Each wavelength and full color speckle is measured across varying distances between simulated DHD.

\section{WAVELENGTH IN DIFFRACTIVE OPTICS}

Previous work has been completed simulating DHDs for speckle reduction at $535 \mathrm{~nm}$ green laser light [6, 7]. Speckle contrast of $0.43( \pm 0.02)$ was achieved at the single wavelength. Diffusers were manufactured based on this simulated design and tested using a diode-pumped solid state (DPSS) green laser. The simulated and actual speckle images from the DHD are shown side-by-side in Figure 1. Physical bench top testing resulted in a minimum speckle contrast measurement of $0.40( \pm 0.08)$ [7]. A grayscale $640 \times 480$ detector with $7 \mu \mathrm{m}$ pixels was used with a $25 \mathrm{~mm}$ projection lens. Laser beam power was kept constant at $0.1 \mu \mathrm{m}$ to decrease the possibility of light saturation disrupting speckle measurements. More specific details can be found within [6,7]. While the speckle reduction is not as low as other proposed reduction systems, the DHD do not require any rotation or vibration creating a 


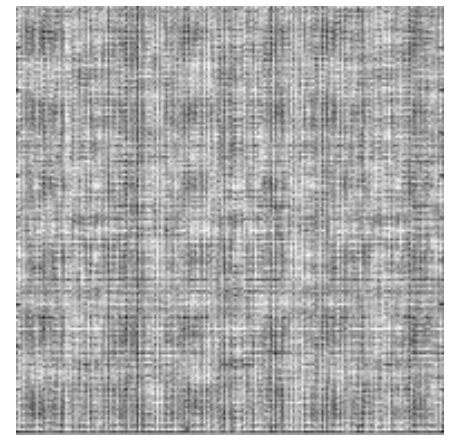

(a)

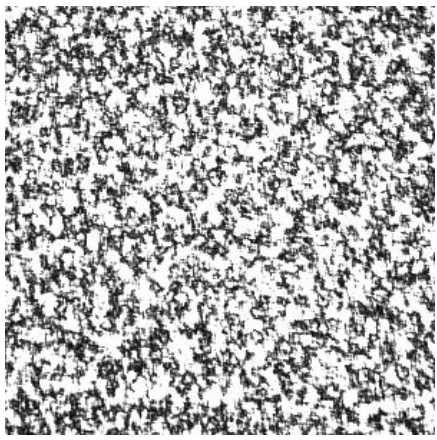

(b)
FIG. 1 Simulated a) and experimental b) speckle images from DHDs with $535 \mathrm{~nm}$ laser light.

completely stationary subsystem ideal for handheld electronic devices.

Diffractive diffusers can be designed to have up to 32 varying phase levels but all diffractive optics can still be described by the grating equation. The grating equation at normal incidence, $d \sin (\theta)=m \lambda$ describes diffracted light normally incident on the diffuser [8]. The order of the diffracted light $(m)$ is given by output angle $\theta$, with $d$ being the period of the grating and $\lambda$ as the wavelength. Keeping the period of the grating constant but changing the wavelength will alter the output angle at each order. This will cause a change in the speckle spot size for differing wavelengths should the distance between grating and imaging plane stay the same. The importance of spectral dispersion is critical when attempting to combine red, green, and blue while reducing speckle contrast across each wavelength using a single optical element. The consequence of the relationship between diffraction angle and wavelength is that the three speckle images combined do not have overlapping speckle spots. The DHDs work in conjunction with an image projection device whether it is MEMS based, line scanning or a micro-lens array. The speckle pattern will be projected onto this device prior to the final image being displayed. Choosing the right projection lens and device pixel size parameters the non-overlapping polychromatic speckle from the DHDs will allow for spatial averaging across multiple speckle spots. The observer or detector perceives an effective smoothing as dark pixels are eliminated and speckle contrast is reduced.

\section{MEASURING RGB SPECKLE CONTRAST IMAGES}

Two possible methods exist to setup diffusers for operation in an RGB laser configuration. Diffusers can be placed after the lasers have been combined and aligned together and then focused with a single projection lens as shown in Figure 2. A consequence of this is that the phase shift is not consistent across all three wavelengths. A second setup includes separate diffuser(s) for each laser prior to combination and alignment. Including multiple diffusers allows you to optimize the phase shift specifically for each wavelength, however space needs are increased. Different projection lenses may be required to keep the output speckle image consistent in size for each wavelength. Due to the complexity of using multiple dif-

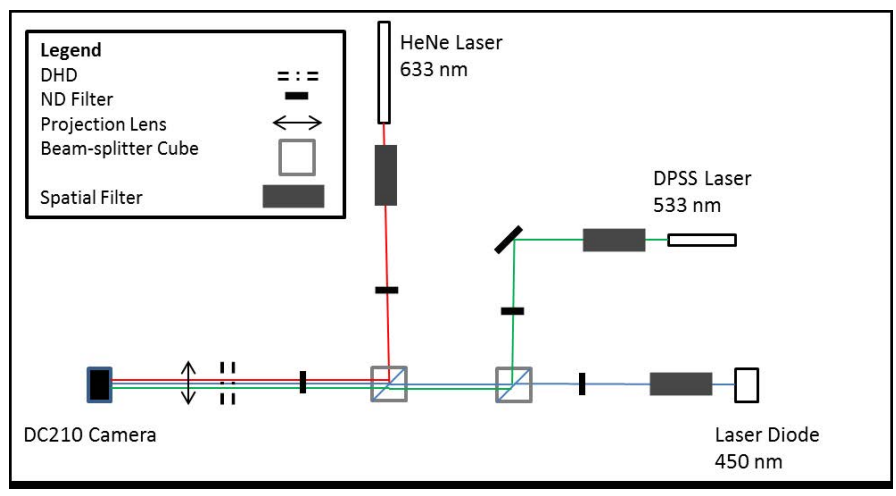

FIG. 2 Multi-wavelength speckle setup using a single DHD.

fusers a compact single diffuser design was chosen for RGB applications.

Multi-wavelength speckle contrast was measured by creating a Fourier optics based simulation to propagate the laser through the diffuser onto a detector [7]. Theoretical calculations for monochromatic DHD result in a contrast of 0.35 . Introducing two additional non-interfering speckle patterns from DHD (two diffusers) adds 8 additional degrees of freedom resulting in a total speckle reduction of 0.13 [1]. However, color detectors do not capture differing wavelengths across a single pixel but separate into red, green and blue. Speckle contrast is then measured by the average across three separate intensity images resulting in a theoretical speckle contrast of 0.11 . Grayscale detectors measure multi-wavelength amplitude over a single pixel creating a single contrast pattern. Unity normalization is performed to ensure comparison of both simulated detection output images.

Each detection method was simulated for full color speckle patterns in order to compare with bench top results using a grayscale camera for image capture. Laser wavelengths of $635 \mathrm{~nm}, 535 \mathrm{~nm}$, and $450 \mathrm{~nm}$ were used to coincide with common laser wavelengths. Grayscale detection resulted in a speckle contrast of $0.19( \pm 0.03)$ while color detection resulted in a speckle contrast measurement of 0.18 . Figure 3 demonstrates the output results for each of the detection methods as well as the averaged RGB image.

Optical bench results are in line with the setup from Figure 2 and so the simulation must account for only a single physical diffuser. An etched diffuser will have fixed phase, $\Delta \phi$, levels based on Eq. (1) [9].

$$
\phi(\lambda, d, n)=\frac{2 \pi(n-1) d}{\lambda}
$$

For fused silica $n$ is 1.4548 at $\lambda=535 \mathrm{~nm}$. Given a known phase difference the etched phase level depth $d$ is determined. The etch depth will correspond to differing phase values at differing wavelengths resulting in a slight phase change difference for each wavelength incident on the diffuser. For RGB applications there is $a+\pi / 10$ phase shift at $635 \mathrm{~nm}$ and $\mathrm{a}-\pi / 10$ phase shift at $450 \mathrm{~nm}$. Adjusting the simulation by simply altering the phase screen of propagation for each wavelength can account for the difference resulting in speckle contrast measurement of $0.24( \pm 0.05)$ and $0.22( \pm 0.05)$ for grayscale and color detection, respectively. Although slight phase adjustments do 


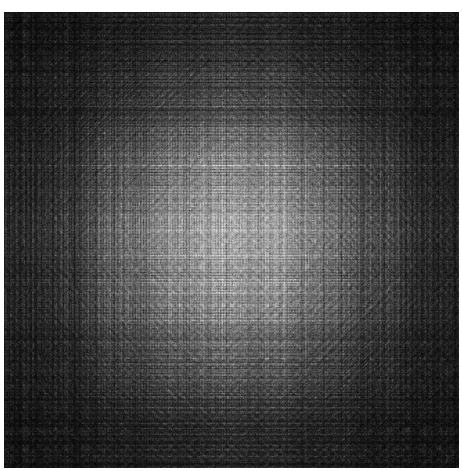

(a)

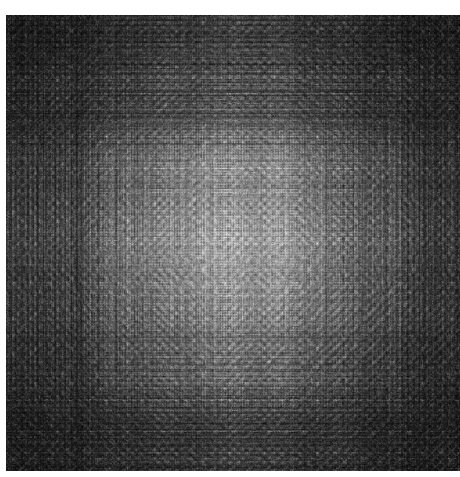

(b)

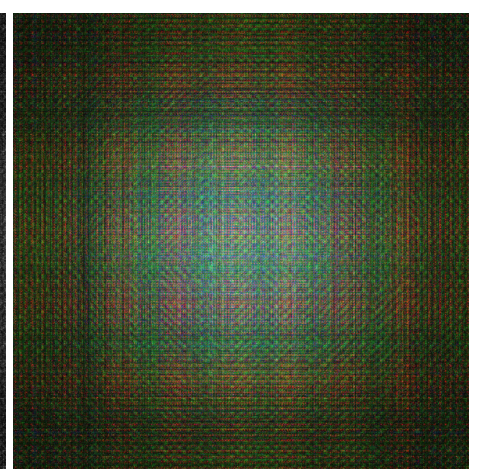

(c)

FIG. 3 Simulated RGB DHD in a) grayscale, b) averaged RGB image, c) full color RGB image.

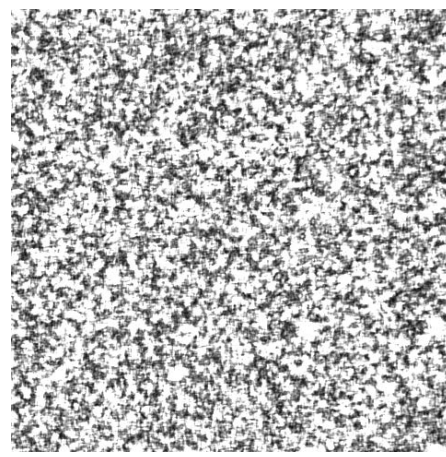

(a)

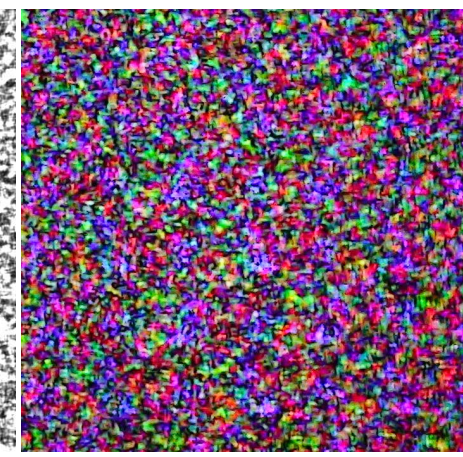

(b)
FIG. 4 Experimental a) grayscale and b) RGB speckle image.

have an impact on the final speckle contrast the values will be used for comparison with the bench top results.

Experimental results for a three laser speckle image were analyzed for speckle contrast reduction. All three lasers maintain the same beam size and intensity at incidence with the diffuser. A Helium Neon laser (at $632.8 \mathrm{~nm}$ ) has a linewidth of $0.002 \mathrm{~nm}$; the $535 \mathrm{~nm}$ laser a linewidth of $0.0001 \mathrm{~nm}$; and the $450 \mathrm{~nm}$ laser has a linewidth of $2 \mathrm{~nm}$. The laser linewidth is inversely related to the coherence length of the laser diode so the $450 \mathrm{~nm}$ laser diode has a much shorter coherence length than either the 535 or $632 \mathrm{~nm}$ lasers. A Thorlabs DC210 CCD grayscale camera was used to capture the speckle images with a frame rate of $30 \mathrm{~Hz}$ and a resolution of $640 \times 480(7 \mu \mathrm{m}$ pixels). The spectral response of the camera was examined in order to keep the irradiances equal across all three wavelengths. The responsivity of the CCD detector at 450 and $632 \mathrm{~nm}$ is $30 \%$ less than the response of the detector at $535 \mathrm{~nm}$. The power levels of the laser were adjusted to ensure measurement accuracy. A speckle contrast value of $0.25( \pm 0.08)$ was measured. Figure 4(a) shows the final grayscale bench top image captured with Figure 4(b) showing the same bench top image except with RGB detection. RGB detection was done with a generic Sony color camera. It had a resolution of $2016 \times 1134$ with a frame rate of $30 \mathrm{~Hz}$.

It is important to highlight that while contrast values are used to quantify the visual disturbance caused by speckle, the "amount" of speckle each observer views is relative and variable. This was proved by [10] through human observations across multi speckle colors and speckle contrast levels. In addition, the final speckle patterns shown are intermediate im- ages and do not represent the speckle that would be viewed by a human observer. It should also be noted that the speckle in Figure 4(a) \& 4(b) are observed from an image and not real time on a stationary background which will alter the perception of the degree of visual disturbance.

\section{SEPARATION OF DIFFUSERS}

Speckle contrast values were slightly above the expected theoretical values. This can be accounted for if we realize that with two diffusers there is a distance between the diffusers which can be altered. Simulations were conducted that analyze how the separation between the diffusers affects the speckle contrast. Separation distance of the DHD were compared across the three wavelengths separately and with all three wavelengths combined using both grayscale and color detection. The individual wavelength results were simulated assuming they used a single DHD that had a $\pi / 2$ phase change at $535 \mathrm{~nm}$. Figure 5(a) shows the separation distance of the DHD versus wavelength at 635,535 and $450 \mathrm{~nm}$.

The x-axis of Fig. 5a is given in unit steps which are $10 \mathrm{~m}$ per step. The distance range is $1-5 \mathrm{~mm}$. Each wavelength curve had its own minimum at which speckle contrast is least. This information allows for monochromatic speckle reduction optimization and can be used to roughly determine the best location for DHD separation with all three wavelengths combined. This distance value can be confirmed by simulating all three diffusers identically across a grayscale and RGB detector. Fig. $5 \mathrm{~b}$ demonstrates both detection method curves for an RGB speckle image.

The speckle contrast value can be further reduced by $5 \%$ by increasing the separation distance between the diffusers. Figure 5(a) explains the reason behind the change is because of the dramatic decrease in speckle for the $450 \mathrm{~nm}$ wavelength diffuser curve. It is of interest that the 535 and $635 \mathrm{~nm}$ curves match one another in shape with the main difference being a $6-8 \%$ contrast increase yet the $450 \mathrm{~nm}$ curve starts quite high and drops until it appears to level with the $535 \mathrm{~nm}$ line. Increasing the separation distance of the diffuser spreads out the speckle spots from the first diffuser to the second. The $450 \mathrm{~nm}$ wavelength, at the lowest visible wavelength, has the smallest diffractive output angle. The smaller angle of the output intensity pattern means more condensed speckle spots being projected onto the $2^{\text {nd }}$ diffuser. A shorter separation dis- 


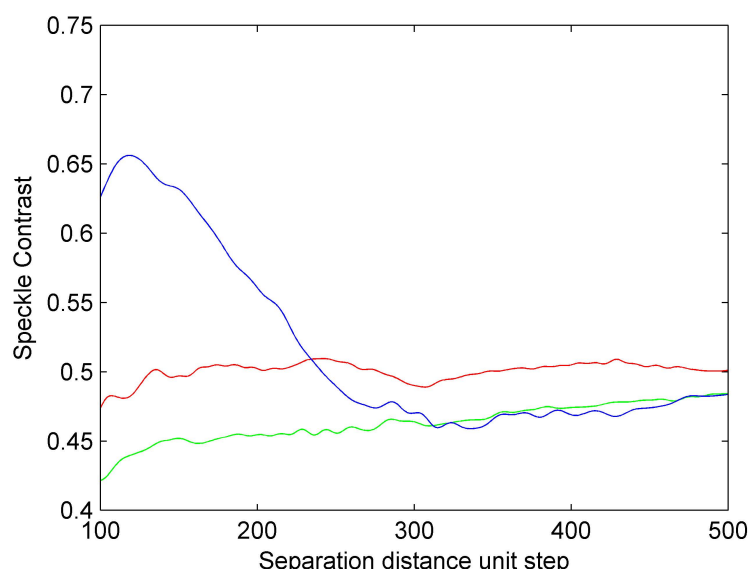

(a)

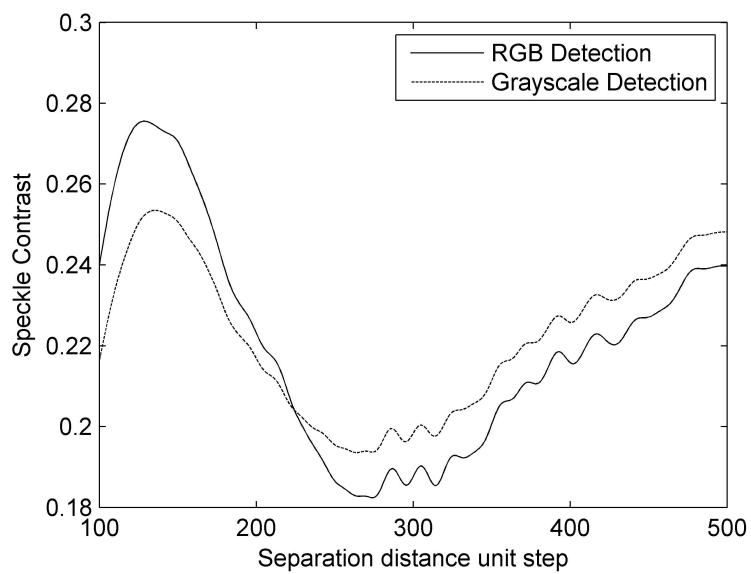

(b)

FIG. 5 Separation distance between the DHD for all three wavelengths: a) at $635 \mathrm{~nm}$, $535 \mathrm{~nm}$ and $450 \mathrm{~nm}$; b) Full color using grayscale and RGB detection.

tance between the diffusers means $2^{\text {nd }}$ diffuser is essentially under-filled. As separation distance increases the speckle pattern incident on the $2^{\text {nd }}$ diffuser gets larger and the contrast decreases. This identifies a very obvious speckle contrast minimum that can be achieved by setting the diffuser spacing. This allows a speckle contrast value of 0.18 to be realized.

\section{CONCLUSION}

Polychromatic speckle contrast has been reduced to $18 \%$ using a single static dual-Hadamard diffuser system. Three wavelengths of 635,550 and $450 \mathrm{~nm}$ were used in speckle contrast measurements complying with standard RGB image projection requirements. Contrast results were compared using grayscale and RGB detection to satisfy inconsistent measurement methods when attempting to measure 3 dimensions of speckle contrast instead of one. In addition, the spacing between Hadamard diffusers was optimized to be used with RGB laser sources individually and together. The dualdiffuser is perfectly suited for integration in a hand-held electronic device without the need for any rotating or vibrating components while still reducing the speckle contrast of the projected image by over $80 \%$.

\section{References}

[1] J. W. Goodman, Speckle phenomena in optics: theory and applications ( $1^{\text {st }}$ edition, Roberts at Company, Englewood, 2007).

[2] J. W. Goodman, "Some fundamental properties of speckle," J. Opt. Soc. Am. 66, 1145-1150 (1976).

[3] G. Ouyang, M. N. Akram, K. Wang, Z. Tong, and X. Y. Chen, "Laser speckle reduction based on angular diversity induced by Piezoelectric Benders," J. Europ. Opt. Soc. Rap. Public 8, 13025 (2013).

[4] T. K. T. Tran, S. Subramaniam, C. P. Le, S. Kaur, S. Kalicinski, M. Ekwinska, E. Halvorsen, et al., “Design, modeling, and characterization of a microelectromechanical diffuser device for laser speckle reduction," J. Microelectromech. Syst. 23, 117-127 (2014).

[5] W. Gao, S. Ma, and X. Chen, "Speckle reduction in line scan laser display system by static diffuser with 2D binary code," Chin. Opt. Lett. 10, S10301 (2012).

[6] W. Thomas, and C. Middlebrook, "Speckle reduction in imaging projection systems," Opt. Photonics J. 2, 338-343 (2012).

[7] W. Thomas and C. Middlebrook, "Non-moving Hadamard matrix diffusers for speckle reduction in laser pico-projectors," J. Mod. Opt. 61, S74-S80 (2014).

[8] C. Palmer, and E. Loewen, Diffraction grating handbook (6 $6^{\text {th }}$ edition, Newport Corp., Rochester, 2005).

[9] C. A. Mack, Field guide to optical lithography (SPIE Publications, Bellingham, 2006).

[10] S. Roelandt, Y. Meuret, G. Craggs, G. Verschaffelt, P. Janssens, and $\mathrm{H}$. Thienpont, "Standardized speckle measurement method matched to human speckle perception in laser projection systems," Opt. Express 20, 8770-8783 (2012). 